\author{
Military Technical College \\ Kobry El-Kobbah, \\ Cairo, Egypt
}

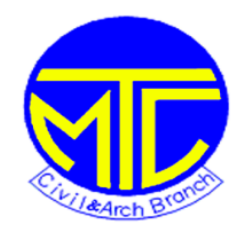

\section{$11^{\text {th }}$ International Conference on Civil and Architecture Engineering ICCAE-11-2016}

\title{
State of the Art Review on Bridges Structural Health Monitoring (Model Testing)
}

\author{
Khaled M. Heiza ${ }^{1}$, Ayman H. Khalil ${ }^{2}$, Omar A. El Nawawy ${ }^{3}$ \\ ${ }^{1}$ Professor of RC Structures, Vice Dean, Faculty of Engineering, Menofia University, Egypt. \\ ${ }^{2,3}$ Professor of RC structures, Faculty of engineering, Ain Shams University, Egypt.
}

\begin{abstract}
Most structural health monitoring methods focus on using dynamic responses to detect and locate damage (i.e. items I and II above) because they are global methods that can provide rapid inspection of large structural systems. These dynamic-based methods can be divided into four groups: (1) spatial-domain methods, (2) modal-domain methods, (3) time-domain methods, and (4) frequency- domain methods. Spatial-domain methods use changes of mass, damping, and stiffness matrices to detect and locate damage. Modal-domain methods use changes of natural frequencies, modal damping ratios, and mode shapes to detect damage.
\end{abstract}

\section{Introduction}

In the frequency domain method, modal quantities such as natural frequencies, damping ratio, and model shapes are identified. The reverse dynamic system of spectral analysis and the generalized frequency response function estimated from the nonlinear auto-regressive moving average (NARMA) model were applied in nonlinear system identification. In time domain method, system parameters were determined from the observational data sampled in time. It is necessary to identify the time variation of system dynamic characteristics from time domain approach if the properties of structural system change with time under the external loading condition. Moreover, one can use model-independent methods or model-referenced methods to perform damage detection using dynamic responses presented in any of the four domains. Literature shows that model independent methods can detect the existence of damage without much computational efforts, but they are not accurate in locating damage.

On the other hand, model-referenced methods are generally more accurate in locating damage and require fewer sensors than model-independent techniques, but they require appropriate structural models and significant computational efforts. Although time-domain methods use original timedomain data measured using conventional vibration measurement equipment, they require certain structural information and massive computation and are case sensitive. Furthermore, frequencyand modal-domain methods use transformed data, which contain errors and noise due to transformation. Moreover, the modeling and updating of mass and stiffness matrices in spatialdomain methods are problematic and difficult to be accurate.

There are strong development trends that two or three methods are combined together to detect and assess structural damages. For example, several researchers combined data of static and modal tests to assess damages. The combination could remove the weakness of each method and check each other. It suits the complexity of damage detection.

\section{LABORATORY AND FIELD TESTING RESEARCH}

Research on modal testing as a health-monitoring tool for civil engineering structures has been ongoing for two decades. The modal testing includes ambient vibration testing and forced 
vibration testing. In ambient vibration testing, the input excitation is not under control. The vibration may be induced be wind, waves, vehicle or pedestrian traffic or any other service loading. The increasing popularity of this method is probably due to the convenience of measuring the vibration response while the bridge is under in-service and also due to the increasing availability of robust data acquisition and storage systems. Since the input is unknown, certain assumptions have to be made. Forced vibration testing involves application of input excitation of known force level at known frequencies. The excitation manners include electro-hydraulic vibrators, force hammers, vehicle impact, etc. The static testing in the laboratory may be conducted by actuators, and by standard vehicles in the field-testing. A brief description of the laboratory and field-testing research on the damage assessment is given below.

Among the earliest uses, Douglas and Reid [i] conducted modal tests on a five-span reinforced concrete bridge to: (1) determine the transverse modal characteristics of the bridge to help understand the bridge response to earthquakes and (2) experimentally evaluate the soil-structure interaction behavior. The authors indicated that modal characteristics are potential tools for system identification of bridge structures. At the University of Missouri, Salane and Baldwin [ii] monitored the modal characteristics of a bridge model as well as a three-span bridge during fatigue tests. A common trend was that frequencies of vibration decreased and the mode shapes changed Research on modal testing, as a global inspection method for civil engineering structures has been ongoing for two decades. A common trend was that frequencies of vibration decreased and the mode shapes changed as the test progressed indicating deterioration. However, reductions in the modal frequencies were small compared to changes in the mode shapes. Thus, the authors commented that changes in specific mode shapes were the best indicators for damage locating. The overall results indicated that vibration signature monitoring could have application to prevent catastrophic failures of bridge structures.

Bakht and Jaeger [iii] summarized the valuable lessons learned from static and dynamic testing of more than 225 bridges in Ontario, Canada. They found that: (1) slab-on-girder bridges are stiffer than the corresponding calculated values; and (2) the floor systems of steel truss bridges may contribute substantially of the combined stiffness of the structure. In most cases, the actual loadcarrying capacities are higher than those from calculations.

Kennedy and Grace [iv] investigated the dynamic and fatigue response of continuous composite bridges with pre-stressed concrete slabs. Four 'l4-scale models of continuous composite bridges were tested. It was shown that pre-stressing the concrete deck slab in the vicinity of the pier supports eliminated transverse cracking of the slab, enhanced the natural frequencies, and increased the fatigue life as well as the ultimate load-carrying capacity.

Mazurek and DeWolf [v] conducted ambient vibration tests of a two-span aluminum plate-girder bridge in the laboratory. They used low-mass vehicular excitation and found that the ambient vibration method provided approximately the same resonant frequencies and mode shapes as those used modal analysis.

Hearn and Testa [vi] applied a perturbation method to structural inspection through vibration monitoring. They found that changes in modal frequency and damping can be good damage indicators; and demonstrated the effectiveness of this method by testing a welded four-member steel frame with progressive cracks. They found that modal parameters (except mode shapes) could be used effectively to detect damage in these test structures.

Hogue, Aktan, and Hoyos [vii] carried out an impact excitation test on local region of a 262-meter long, pre-stressed, pre-tensioned concrete girders bridge. Modal parameters except for damping ratios were identified, then the mass matrix was estimated, and then the flexibility matrix was derived. A static test was conducted to validate the dynamic test-based identification.

Pandey and Biswas [viii] used a simple supported W12x16 beam for experimental verification. The beam had a splice at the mid-span. Damage was simulated by opening bolts from the splice plates. Thirty-three measurement points were marked up on the top of the beam. They 
demonstrated the effectiveness of using changes in the flexibility matrix in detecting and locating damages.

Salawu and Williams [ix] conducted full-scale forced-vibration tests before and after structural repairs on a multi-span reinforced concrete highway bridge. A hydraulic actuator was used to excite the bridge, and four servo-accelerometers were used to measure the bridge response. They found that: (1) the natural frequencies did not change significantly due to structural repairs; and (2) the modal assurance criterion and the coordinate modal assurance criterion values were found to be good indicators of the presence and location for condition assessment of the bridge. The modal analysis gave an indication of the location of the repairs. They recommended more experimental work for condition assessment of the bridge. In a similar study, the same authors [x, $x i$ ] investigated the effects of bearing replacement on the modal characteristics of a six-span reinforced concrete bridge as that in their previous investigations. The author used frequency shifts in addition to mode shape derivatives to examine damage presence. Results indicated that bearing replacement did not significantly affect the natural frequencies; however, there was a marginal change in mode shape derivatives. Further, results showed that environmental conditions had minimal effect on the modal properties.

Krishnan et al. [xii] examined the changes in the natural frequencies of a T-girder bridge as results of a four-phase repair and strengthening program. The repair included adding an overlay of structural concrete, injecting the flexural cracks, external post tensioning, and adding a bottom slab. Laboratory controlled tests were conducted to obtain the frequency response function plots. Frequency shifts successfully indicated all of these stages, but with different levels of accuracy.

Farrar et al. [xiii] has done extensive testing of damage detection methods on the Alamoso Canyon Bridge in New Mexico. Both forced and ambient vibrations were measured using a very dense array of accelerometers. Modal parameters were extracted using linear signal processing techniques and compared with those calculated using a detailed finite-element model. One of the important outcomes of this experiment was a study of the variability of various modal parameters and an attempt to quantify the statistical significance of parameter changes.

Doebling, Hemez, et al. [xiv] employed vibration tests of a scale suspended truss to prove that a mode selection strategy based on maximum modal strain energy produces more accurate update results than a strategy based on minimum frequency. The truss consisted of eight bays, with a span of $4 \mathrm{~m}$. Eight lumped masses connected to it. They made the truss as an appropriate representation of large spacecraft structures such as International Space Station Alpha. Similar tests were also conducted by Kosmatka and Rides [xv]. Their experimental results showed that the proposed method in conjunction with residual forces and a weighted sensitivity analysis could accurately predict the location and severity of stiffness as well as any changes in mass for different damage scenarios.

Lenett et al. [xvi,xvii] examined two basic assumptions related to modal testing and its application to bridges: (1) linearity and (2) time invariance. The authors commented that a bridge should satisfy these conditions in order to validate the results of a modal test. Modal tests were conducted on a bridge with several sources that may cause non-linearity such as the reinforced concrete deck slab and the asphalt overlay. Linearity checks were performed by two means. First, reciprocity of frequency response function developed through concrete-asphalt measurements (impact on concrete and response of asphalt and conversely) was checked. Second, frequency response functions resulting from different levels of impact force (varying drop height of the impact hammer) were compared. It was verified that the bridge response is linear within the frequencies of interest (less than $30 \mathrm{Hertz}$ ). On the other hand, the bridge showed a time-variant behavior during testing. The authors suggested that testing be conducted in as short a time as possible and at a particular time of the day. Different methods to post-process the raw data, force input and acceleration response time histories were summarized. 
Haritos and Chalko [xviii] described the modal test procedure for bridges in Australia, where frequency response functions were obtained at closely spaced points (5 ft apart) on a reinforced concrete three-span bridge with span length of approximately $35 \mathrm{ft}$. Structural defects, such as loss of flexural rigidity from the assumed fixed condition at the abutments, were detected by inspecting the mode shapes.

Sennah and Kennedy [xix] presented the dynamic characteristics results from an extensive parameter study on the free vibration and forced vibration of simply supported, two or three-span continuous, straight and curved, composite multi-cell box girder bridges. These results are helpful to the damage detection study on the composite bridges by vibration modal testing.

Chen, Yang, et al. [xx] tested a full-scaled beam both statically and dynamically. They focused on the correlation between frequency of a structure and degree of damage. Test results have confirmed that the frequency of the beam itself depends on the load history while that of the beam plus sufficient preloads can be identified independently. This was because pre loads can keep cracks open so that the cracked beam vibrates in a linear fashion.

Lee and Liang [xxi] have developed a portable system integrated with necessary computer software and measurement hardware, such as sensor and data-acquisition systems for bridge condition assessment. They have established the viability of using this system through a 1:6 down scaled model bridge. The bridge model is a typical 1-meter wide concrete slab supported by three single span parallel steel girders with a span of 2.50 meters. On the slab, a total 16 measurement points were chosen. Ambient excitations were used for the modal testing, which is generated by pulling a model car along the bridges. The results showed that the energy transfer ratio (ETR) is a good indicator of structural damage. Ambient and impact tests on three full scaled bridges were also carried out. No real damage existed in these bridges, therefore no notable changes presented in the measured parameters.

Aktan, et al. [xxii,xxiii] proposed a coordinated, multi-disciplinary approach that integrated field, theoretical and laboratory research for solving large system identification problems. A 3-span steel bridge testing in field and its physical model testing in the laboratory were conducted for the damage detection. The studies indicated that modal flexibility provided a relevant/reliable measure of structural state. Also many experiences, observations, as well as challenges were summarized.

Huang, Yang, et al. [xxiv] presented a simple and effective procedure for conducting the free vibration test on highway bridges. The feasibility of the procedure was demonstrated in identification of the dynamic properties of a three-span box-girder concrete bridge Using ITD technique. Chaudhary, Abe, et al. [xxv] utilized the strong seismic records in the 1995 Kobe earthquake to examine the performance of various components of two base-isolated bridge, based on the method of parameter identification. It is shown that the identified system parameters could reveal useful information about the performance of the isolation system as well as about different substructure components.

Hwang, Jernigan and Lin [xxvi] presented a procedure for the evaluation of the expected seismic damage to bridges and highway systems. The bridge damage states considered were no/minor damage, repairable damage, and significant damage. Given an earthquake with a moment magnitude of 7.0, the expected damage to bridges and highway systems was determined. The results could be used to prioritize bridges for retrofitting, to prepare a pre-earthquake preparedness plan, to develop a post-earthquake emergency response plan, and to assess the regional economic impact from the damage to highway transportation systems. Haritos, N. [xxvii] introduced the several years of experience in the dynamic testing on bridge superstructures for the structural system identification at the university of Melbourne, Australia. They developed a modal testing package by using simplified experimental modal analysis and time -domain identification method. A number of bridges were tested and analyzed using this package.

Piombo, Fasana, et al. [xxviii] described the dynamic tests performed on a simply supported bridge with a span of 20 meter in Northern Italy under traffic excitation. The acceleration data had been 
used for the identification of the natural frequencies, viscous damping ratios and mode shapes of the bridge. Modal parameters had been extracted using the wavelet estimation technique. This work represented the first attempt in using the wavelet estimation technique directly on transient data and nor on the impulse response estimates obtained via the random decrement technique.

Kim and Bartkowicz [xxix] designed and built a ten-bay hexagonal truss to simulate the current space station truss sections. Modal tests were performed on this laboratory structure partially instrumented with 96 accelerometers in several damage conditions. A time-domain modal identification technique was used to extract frequencies and mode shapes from the test data. A two-step damage detection approach was developed and showed promise for large structures with limited instrumentation. Because the proposed approach is a global nondestructive evaluation method which uses vibration measurements and, therefore, it is limited to identifying structural damages. Several damage cases were determined to be unidentifiable.

Halling, Muhammad, and Womack [xxx] carried out seven forced vibration tests on an isolated single span of a freeway overpass structures. The work focused on horizontal vibration utilizing an eccentric mass shaking machine and the data was collected with an array of accelerometers. Finiteelement models were created to simulate the structure. It was shown that the structural parameters optimized from the algorithm could be used to identify the estimated location and intensity of the damage or retrofit of the structure at each state.

Peeters and Roeck [xxxi] conducted one-year monitoring of the Z24-Bridge in Switzerland under ambient vibrations. They presented a method to distinguish normal Eigen-frequency changes form the environmental effects, such as wind and temperature, on damage events. Further research will focus on a description of the non-linear behavior so that safety statements about bridge in cold period will be made.

From above, we can distinguish that (1) the models in the laboratory are mainly beams, columns, truss and/or frame structures; (2) the location and severity of damage in the models are determined in advance; (3) the testing has demonstrated lots of performances of damage structures; (4) the field-testing and damage assessment of real bridges are more complicated than the models in the laboratory; and (5) the correlation between the damage indicator and damage type, location, and extent still needs further improvement.

\section{ANALYTICAL DEVELOPMENT}

The bridge damage diagnosis and health monitoring are both concerned with two fundamental criteria of the bridges, namely, the physical condition and the structural function. In terms of mechanics or dynamics, these fundamental criteria can be treated as mathematical models, such as response models, modal models and physical models. Instead of taking measurements directly to assess bridge condition, the bridge damage diagnosis and monitoring system evaluate these conditions indirectly by using mathematical models.

The damage diagnosis and health monitoring are active areas of research in recent years. For example, numerous papers on these topics appear in the proceedings of International Modal Analysis Conferences (IMAC) each year, in the proceedings of International Workshop on Structural Health Monitoring (once of two year, at Stanford University), in the proceedings of European Conference on Smart materials and Structures and European Conference on Structural Damage Assessment Using Advanced Signal Processing Procedures, in the proceedings of World Conferences of Earthquake Engineering, and in the proceedings of International Workshop on Structural Control, etc..

There are several review papers to be referenced, for examples, Housner et al. [Error! Bookmark not defined.] provided an extensive summary of the state of the art in control and health monitoring of civil engineering structures. Salawu [xxxii] discussed and reviewed the use of natural frequency as a diagnostic parameter in structural assessment procedures using vibration monitoring. Doebling, Farrar, et al. [Error! Bookmark not defined.,xxxiii] presented a thorough 
review of the damage detection methods by examining changes in dynamic properties. Zou, Tong, and Steven [xxxiv] summarized the methods of vibration-based damage and health monitoring for composite structures, especially in delamination modeling techniques and delamination detection.

\section{Signature Analysis and pattern Recognition Approaches}

A modal model is characterized by a set of modal parameters, which can be extracted from response model by modal testing techniques. Traditionally, the major modal parameters are natural frequencies, damping ratios, and mode shapes. The modal model can be also used as a vibrational signature. For example, in mechanical engineering condition monitoring of rotating equipment is typically based on looking for signature changes in a power spectrum of the measured vibrations. The same nonparametric approach could be used for bridges, but it is more typical to use identified modal parameters to provide the signature characterizing the structure. In order not only to detect damage but to also locate its position, observed changes in the signature must be compared with a database of possible changes and the most likely change may be selected. This is a type of pattern recognition where the database of "pattern" is generated by analyzing various damage scenarios or "failure modes". The representative researches on damage detection through a modal model are listed briefly in the following.

One approach to detect damage has been to use changes in the modal frequencies. With fiberreinforced plastics, Adams, et al. [xxxv] demonstrated that damage could be detected from a decrease in natural frequencies and in an increase damping. Biswas, Pandey, and Samman [xxxvi] performed experiments on a highway bridge and demonstrated that the decrease in natural frequencies can be used to detect the presence of damage. Capecchi and Vestroni [xxxvii] addressed the problem of understanding when it is sufficient to measure and use only natural frequencies, thus avoiding the need to measure modal shapes in vibration beams, or beam systems. The identification procedure was based on the minimization of an objective function that accounts for the difference between the analytical and experimental quantities. Further study demonstrated that the observed changes in natural frequencies, especially the changes in fundamental natural frequencies, were unable to determine the location of crack damage (Casas, et al. [xxxviii]). This occurs because a certain amount of damage at two different locations may produce the same amount of frequency change.

Sensitivity analysis has been proposed to improve the sensitivity of natural frequency change to the structural damage (Hearn and Testa, [xxxix]). The basic idea behind this was to compare the frequency changes obtained from experimental data collected on the structural with the sensitivity of the modal parameters obtained from an analytical FE model of the structure. Accuracy of sensitivity-based methods is dependent on the quality of the FE model used to computer the sensitivities. It should be kept in mind that obtaining an accurate analytical model in itself remains a difficult task. The uncertainties of analytical model may influence the results of damage detection.

Results from some experimental and numerical studies suggested that the lower vibration modes would probably be suited for damage detection. Using the information from the mode shapes, Stubbs et al. [xl] reported a method to localize damage by using the pattern recognition method. They studied a beam model with known mode shapes, and then generated mode shapes at any location using interpolation. The location of damage compared fairly well with FE analysis. Finally, they applied this method to the real bridges including a 50-m-plate girder bridges and a two-span simply supported truss bridges (the length of each span is approximately 70 meters), and in general concluded that the method can accurately locate damage though the damage pattern was not quite distinctive.

The combination of different modal parameters, especially the combination of natural frequency and mode shapes has been used by several researchers. Mazurek and DeWolf [v] found that crack propagation in a beam can cause substantial shifts in certain frequencies and mode shapes can be 
used to locate the damage. With the help of analytical beam models, Pandey et al. [xli] demonstrated the use of changes in the curvature mode shapes to detect and locate damage. Wahab and Roeck [xlii] introduced a damage indicator called" curvature damage factor", in which the difference in curvature mode shape for all modes can be summarized in one number for each measured point. They applied the techniques to a real pre-stressed concrete bridge, named Z24, in Switzerland.

Another method for examining damage in structures is to investigate mode shapes. The Modal Assurance Criteria (MAC), $\operatorname{MAC}\left(\left\{\phi_{i}^{\mathrm{X}}\right\},\left\{\phi_{j}^{\mathrm{Y}}\right\}\right)=\frac{\left(\left\{\phi_{i}^{\mathrm{X}}\right\}^{\mathrm{T}}\left\{\phi_{\mathrm{j}}^{\mathrm{Y}}\right\}\right)^{2}}{\left(\left\{\phi_{\mathrm{i}}^{\mathrm{X}}\right\}^{\mathrm{T}}\left\{\phi_{\mathrm{i}}^{\mathrm{X}}\right\}\right)\left(\left\{\phi_{\mathrm{j}}^{\mathrm{Y}}\right\}^{\mathrm{T}}\left\{\phi_{\mathrm{j}}^{\mathrm{Y}}\right\}\right)}$, and the Coordinate Modal Assurance Criteria (COMAC), $\operatorname{COMAC}\left(\{\mathrm{X}, \mathrm{Y}, \mathrm{q})=\frac{\left(\sum_{\mathrm{i}=1}^{\mathrm{N}}\left(\phi_{\mathrm{iq}}{ }^{\mathrm{x}}\right)\left(\phi_{\mathrm{iq}}{ }^{\mathrm{Y}}\right)\right)^{2}}{\sum_{\mathrm{i}=1}^{\mathrm{N}}\left(\phi_{\mathrm{iq}}{ }^{\mathrm{X}}\right)^{2} \sum_{\mathrm{i}=1}^{\mathrm{N}}\left(\phi_{\mathrm{jq}}{ }^{\mathrm{Y}}\right)^{2}}\right.$ are the two most commonly used parameters to detect mode shape changes, with $\left\{\phi_{\mathrm{i}}^{\mathrm{X}}\right\}=$ shape vector of Mode $\mathrm{i}$ of the structure in State $\mathrm{X},\left\{\phi_{\mathrm{j}}^{\mathrm{Y}}\right\}=$ shape vector of Mode $\mathrm{j}$ of the structure in State $\mathrm{Y}$, $\mathrm{q}=$ location where COMAC value is computed, $\mathrm{N}=$ number of modes utilized in computing COMAC values, and $\phi_{\mathrm{iq}}{ }^{\mathrm{x}}=$ ordinate of Mode $\mathrm{i}$ at location $\mathrm{q}$ in state $\mathrm{X}$. Generally, MAC is used to compare similar shapes from different states, i.e., $\mathrm{i}=\mathrm{j}$ and $\mathrm{X} \neq \mathrm{Y}$ in Equation 4, and was first introduced in 1982 to compare modes from an intact Space Shuttle Orbiter body flap and those from the same flap after acoustic loading [xliii]. In that case, the compared states were intact and damaged, respectively; comparatively, they may be experimental and theoretical, mainly, to measure how close a theoretical model to the actual structure. MAC values close to one indicate high correlation and a MAC value of one indicates that the compared modes are identical. MAC values are also used to compare dissimilar mode shapes, i.e., $\mathrm{i} \neq \mathrm{j}$ in Equation 4, primarily to ensure orthogonality among different modes. Orthogonal modes have zero (or very small) MAC values. COMAC value measures the correlation between the ordinates of two sets of mode shapes at a given location. As with MAC, the two sets may be (intact, damaged) or (experimental, theoretical). At a given location, close-to-one COMAC values indicate high correlation between the compared sets. On the other hand, small COMAC values indicate drastic differences (i.e., damage presence in case of comparing damaged to intact mode shapes). Another combination in terms of natural frequencies, mode shapes, and modal assurance criteria (MAC) was employed by Alampalli, Fu, and Aziz [xliv, xlv] and Khalil [xlvi]. They concluded that natural frequencies should be used to detect damage, and mode shapes and MAC values can be further used to identify damage locations.

Zhang, Schulz, and Ferguson [xlvii] employed the transmittance functions (TFs) and the sensoractuator system to detect, locate and assess damages on a composite beam. Further work was underway to use sequential TFs to detect damage on large panel and blade structures using a dense pattern of measurements formal scanning laser Doppler vibrometer.

This kind of vibrational signature analysis has been proven to be successful in localizing damage. However, it is not sensitive to most types of damage that occur to bridge structures. Model testing and field-testing have shown that the changes of natural frequencies due to local damage are very small, mode shapes (especially higher mode shapes) are sensitive to the changes of local stiffness but it is very difficult to measure them accurately. There are similar problems in other vibration signatures, such as mode shape curvature, modal flexibility, MAC, etc. None of these can provide sufficient information for the detection of both small and large defects. The successful applications of these modal model methods may rely on the development of test techniques and new findings of model-based approaches. 


\section{Model Updating and System Identification Approaches}

System identification (SI) is the process of constructing or updating an accurate mathematical model of a system based on input and output (I/O) observations. Among other applications, SI can be applied to structural health monitoring and damage assessment, e.g., by determining the structural stiffness values and comparing them with previously determined values or originally intended values. Research interest in this subject area has increased steadily over the years. Most of the SI studies in structural engineering have dealt with few degrees of freedom (DOFs) and few unknown structural parameters. In practice, however, modeling of engineering structures often requires the contrary. The difficulty and the computational effort required for convergence increase drastically when the numbers of DOFs and unknowns increase. To this end, various means have been proposed in recent years to tackle the numerical problems generally associated with SI of large systems.

Kim and Bartowics [xlviii, xlix] also developed a two-step damage detection and health monitoring approach for large and complex structures with a limited number of measurements. The first step is initial damage detection, based on the optimal-updating techniques and changes of stiffness. The second is detailed damage detection by the design sensitivity method and linear perturbation theory. Classical SI techniques were used, such as EKF, recursive least squares, instrumental variable and maximum likelihood methods. These methods, in one way or another, search the optimal solution by exploiting the previous solution. Treating the problem as an inverse problem, many classical methods require the use of secant, tangent, or higher-order derivatives of the objective function.

As the system of unknowns grows in size, the numerical difficulty increases and often to the extent that the convergence becomes extremely difficult, if not impossible. Such "exploitation" methods perform point-to-point search and have the danger of converging to local optima. On the other extreme, a random search (e.g. trial-and-error) may be used to explore the entire search space. To overcome one trial solution with another, an error norm has to be defined as a measure of deviation of the estimated response (computed based on the estimated parameters) from the actual (measured) response. The search continues until the error norm is deemed to be small. Such a blind "exploration" strategy is obviously too time consuming for large systems due to huge number of possible combinations. For instance, if there are ten unknowns to be identified and each unknown is divided into 100 discrete values within its search range, there will be a total of $10^{20}$ possible combinations-an astronomical figure to work with even for today's powerful computers.

In this regard, a worthwhile attempt is to employ evolutionary algorithms, which have proved in the last decade to be a powerful search and optimization tool. The main features of these algorithms are that they attempt to imitate living things and are stochastic in nature. There are presently four main approaches, namely, genetic algorithms (GA), evolutionary programming, evolutionary strategies, and simulated annealing. By far the most widely known approach in engineering is perhaps GA. This approach was developed to solve discrete or integer optimization problems as opposed to continuous parameter optimization problems. In the case of parameter identification, this can be tuned into an advantage of controlling the resolution of identified parameters through the (integer) length of the chromosome (number of bits). Koh, Hong, and Liaw [1] conducted a GA search in modal domain of a much smaller dimension than the physical domain. The objective function was defined based on the estimated modal response in time domain and the corresponding modal response transformed from the measured response. This method had been shown to work well in terms of mean error (10-15\%) for a fairly large system with 50 DOF's and 52 unknown parameters. Hao and Xia [li] applied a genetic algorithm with real number encoding to identify the structural damage by minimizing the objective function, which directly compares the changes in the measurements before and after damage. Three different criteria are considered, namely, the frequency changes, the mode shape changes, and a combination of two. A laboratory tested cantilever beam and a frame are used to demonstrate the proposed techniques. Numerical results 
show that the damaged elements can be detected by GA, even when the analytical model is not accurate.

Structural system identification within the linear regions has been well developed and many techniques have been applied to structural damage assessment. However, the question of whether a structure is still linear after the damage remains. This is very important because the dynamical behavior of a nonlinear system can be quite different from those of its associated linear system. Also if the structural system becomes nonlinear after damage, its dynamical characteristics cannot be estimated by using the linear system identification methods. The Fast Fourier transform (FFT) and the least-squares method were used to quantify the damage.

Masri et at. [lii,liii] employed the neural network technique to detect the changes in nonlinear systems. The technique was applied to weakly non-linear multi-degree of freedom systems. One of the advantages of the technique was the ability to determine the response of the non-linear system at any level once its variable modal parameters had been identified at some reference force level. The authors also presented the experimental verification and the application to a representative engineering case.

Lin, Betti, Smyth, and Longman [liv] presented an adaptive on-line parameter identification algorithm based on the variable trace approach for the identification of non-linear hysteretic structures. At each time step, this recursive least-square-based algorithm upgrades the diagonal elements of the gain matrix by comparing the value of the estimated parameter between two consecutive time steps. The effectiveness and efficiency of the proposed algorithm was shown by considering the effects of excitation amplitude, of the measurement units, of larger sampling time interval and of measurement noise. Kerschen and Golinval [lv] investigated the vibrations of a clamped beam for two different kinds of non-linearity. Firstly, the beam showed a non-linear behavior characterized by a piecewise linear stiffness and secondly, the non-linearity came from a bilinear stiffness. They demonstrated the performance of the restoring force surface method and presented both numerical and experimental results. Obviously, the nonlinear system identification will be developed by many researchers in the not too far future.

When performing vibration tests on civil engineering structures, such as bridges, it is often unpractical and expensive to use artificial excitation (shakers, drop weights). Ambient excitation on the contrary is freely available (wind, traffic). This output-only system identification now becomes more and more important [xlvi]. Huang and Liu [lvi] applied a subspace approach cooperating with an instrumental variable concept to evaluate the coefficient matrices of a statespace model. The dynamic characteristics of a structure are determined from the coefficient matrices. The feasibility of the procedure is demonstrated through processing an in-situ ambient vibration measurement of a five-story steel frame, an impulse response measurement of a threespan continuous bridge, and simulated earthquake responses of five-story steel frames from shaking table tests.

In structural system identification, different mathematical models will introduce different explanations on the result of identification even with the same set of input/output data. The model inaccuracy in structural system identification can be categorized into two items: (1) the uncertainty due to nonlinear model; and (2) the completeness of model description (or extract description). Selecting the exact model becomes one of the important issues for identification.

\section{Model updating and Mode Selection}

A common theme in using system identification for structural health monitoring and damage diagnosis is to use a model updating approach. Usually, highly accurate and detailed finite-element models (FEMs) are required to analyze and predict the dynamical behavior of complex structures during analysis and design. Once the finite-element model of a physical system is concentrated, its accuracy is often tested by comparing its modes of vibration and frequency response with those obtained from the physical system. If the correlation between the two is poor, then assuming that 
the experimental measurements are correct, the analytical model must be adjusted so that the agreement between the analytical predictions and the test results is improved. The updated model may then be considered a better representation of the physical structure than the initial analytical model. Any observed local decrease in the stiffness of the model is assumed to indicate the location and severity of damage in the monitored structure. The updated model can subsequently be used with reasonable accuracy to assess the stability and control characteristics and to predict the dynamical responses of the structure. The above process of correcting the system matrices is known as model updating.

The methods for FEM update that are used for health monitoring can be divided into the following major categories: mode flexibility methods, optimal matrix update methods, sensitivity-based matrix update methods, Eigen-structure assignment methods, changes in measured stiffness methods, and combined modal parameters methods, etc.. All of these FEM update techniques require that the user selects a subset of the measured modes to be correlated with the corresponding modes of the FEM. Normally, the first few modes of the structure are used in the FEM correlation because they generally the best identified modes. However, in some situations the higher frequency modes are critical to the location of structural damage, and so it is necessary to include them in the set of modes for FEM correlation. Many modes that are below these in frequency do not undergo significant modification as a result of the damage, so that they contribute to the computational burden without contributing significantly to the location of the damage. The number of modes is limited not only by the computational burden, but also by the inherent ill conditioning and statistical bias associated with large-order update problems. Because of this limit, it is important to have systematic criteria for selecting which modes are most indicative of the structural damage. Doebling, Hemez, et al. [xiv] utilized the MAC, mode selection strategies and FE model update to detect damage. They found that a mode selection strategy based on maximum modal strain energy produced more accurate update results than a strategy based on minimum frequency. Lardies and Larbi [lvii] also proposed a new method for model order selection and modal parameter estimation in time domain. The model selection is still a difficult problem to be studied.

\section{Review of Damage Detection Methods}

FE modeling provides a complete set of analytical and theoretical modal parameters for a structure, but these parameters are usually of uncertain accuracy. The experimental data is accurate to some extent, but incomplete, and also intervolved by the noise. Any method to do modal updating must address the mismatch between the level of information in the detailed analytical FEM and the relatively sparse information. Sohn and Law [lviii] presented a general statistical approach, which treats the uncertainties that arise from measurement noise, modeling error, and possible nonuniqueness in the problem of updating the stiffness distribution and used this approach to multiple damage locations.

Xia, et al. [lix] proposed a statistical method with combined uncertain frequency and mode shape data for structural damage identification. The finite element model is updated by comparing the measured vibration data before and after damage occurs. The effects of uncertainties in both the measured vibration data and the finite element model are considered as random variables in model updating. The statistical variations of the updated finite element model are derived with perturbation method and Monte Carlo technique. The probabilities of damage existence in the structural members are then defined. The results between the calculation and testing show that all the damages are identified correctly with high probabilities of damage existence.

Model updating within a statistical framework appears to be a promising general approach to damage diagnosis and structural health monitoring of large civil structures in view of the inescapable data and modeling uncertainties. But many aspects require further research, including optimal location of sensors, the type of damage, which can be reliably detected and reliably located 
using a giving array of sensors on a structure, strategies for making decisions about possible damage and determining the corresponding probabilities of false alarm and missed alarms, etc..

\section{Damage index Methods}

Alamaplli [xliv] proved that the ratio of the model frequency change between any two models is the function of the damage location only. The ratios were then used as damage indicators, which were calculated from a candidate set of assumed possible damage scenarios. The structural damage was then localized by comparing the predicted ratios with the ratios computed based on measured modal frequencies. Wahab and Roeck [xlii] introduced a damage indicator called "mode curvature damage factor" to detect damage of a real pre-stressed concrete bridge.

The modal flexibility involves functions of both the natural frequencies and mode shapes. Some researchers (for example, Pandey and Biswas [viii]) have found experimentally that modal flexibility can be a more sensitive parameter than natural frequencies or mode shapes along for structural monitoring and damage detection in bridges. They studied theoretically the sensitivity by comparing use of natural frequencies, mode shapes, and modal flexibilities for monitoring. The results demonstrated that modal flexibilities are more likely to indicate damage than either natural frequencies or mode shapes. They discovered the changes of the system natural frequency tend to be small in the early stage of damage, and therefore may be difficult to quantify, even from accurately processed recorded motions. Other difficulties arise from the non-uniqueness in the model representation. Unless the model accounts for the soil-structure interaction, and it has been carefully validated and calibrated, it is very difficult to identify the true causes and sources of observed non-linearity in the response.

Shi, et al. [lx] proposed using the ratio of change in model strain energy in each element as another damage indicator. The approach requires only the elemental stiffness matrix, the analytical mode shapes, and the incomplete measured mode shapes. The effect of analytical mode truncation, incomplete measured mode, and measurement noise in the damage detection were discussed. Results from the modal simulation and experiment with a two-story partial steel frame indicated that the presented method is effective in localizing damage, but it is noise sensitive in the damage quantification to some extent. In addition, Shi et al. presented a sensitivity and statistical-based method to localize structural damage by direct use of incomplete mode shapes. In general, the damage detection strategy is to localize the damage sites first by using incomplete mode shapes, and then detect the damage sites and extent again by using measured natural frequencies, which have a better accuracy than mode shapes.

Wang, et al. [lxi] presented a comparative study of applying various mode-based indices to the structural damage detection of the Tsing Ma suspension bridge with a main span of 1377 meter and an overall length of 2160 meter. Five mode-based damage indices, including coordinate modal assurance criterion (COMAC), enhanced coordinate modal assurance criterion (ECOMAC), mode shape curvature (MSC), and modal strain energy index (MSEI), and modal flexibility index (MFI) were applied respectively for the damage location identification of various simulated damage scenarios in the bridge by 3D finite element method. The numerical simulation results show that the applicability and the performance of each index depend on the damage type concerned. Based on the performance evaluation, the preferred damage indices in accordance with different damage types were recommended.

Ren and Roeck [lxii] proposed a damage identification technique at an element level. The element damage equations have been established through the Eigen-value equations based on changes in frequencies and mode shapes of vibration. Several solution techniques are discussed and compared. Numerical results show that the non-negative least-squares method can lead to satisfactory results in most cases. An experimental program of the reinforced concrete beam under static and dynamic loading was used to demonstrate the identification scheme. 
There may be other damage indices to indicate the locations and extent of damage. For real civil structures, only one damage index may not be enough. Until now, the relationships between damage type and damage index are not clear. A lot of further studied are needed in this area.

\section{Methods From Static Data}

Static parameter estimation is based on measured deformations induced by static loads such as a slowing moving track on a bridge. There are many instances in which static loadings is more economical than dynamic loading. Many applications require only element stiffness for condition assessment. In these cases static testing and analysis can prove simple and more cost effective. Since the natural frequencies, mode shapes, and static responses of a structural system are functions of structural parameters, these parameters may be identified by comparing the dynamic and static characteristics predicted from the mathematical model to those values determined by test.

One of the consequences of the development of damage is the decrease in local stiffness, which in turn results in changes in some of the responses. It is therefore necessary that the dynamic and static characteristics of the structure be monitored for damage detection and assessment. Based on the concept, Oh and Jung [lxiii] propose an improved method that can identify a finite-element model of a structure capable of providing structural characteristics that are consistent with those measured in static and dynamic tests (e.g., the curvature of mode and the static displacement data). The detection of damage in a member with stronger influence on the higher modes is more difficult. Thus, the use of static displacements obtained by a loading condition that simulate higher modes was proposed as a solution to this problem. Jang, et al. [lxiv] used the system identification (SI) method to identify structural parameters in a FEM by minimizing the error between measured and analytical computed responses. A regularization scheme is applied to alleviate the ill-condition of an inverse problem by adding a regularization function to the primary error function. Two different algorithms depending on the type of measured response have been developed to assess damage. Static displacements from static loading and modal data from impact vibration were measured through laboratory experiments on a grid-type model bridge. Damage is simulated by saw-cutting the cross section with various depths and identified as the reduction in the structural stiffness of the elements around the crack. Through the experimental works, the applicability of the SI-based damage assessment algorithms has been rigorously investigated.

Modal updating by finite element method is often used to identify the changes of damage using static testing data. Because the errors caused by finite element model may be greater than changes of damage, the finite element models should be firstly calibrated using the measured modal properties and experimental data. Only the finite element models are reliable, the results from modal updating by finite element methods are valuable.

\section{Neural Networks Approaches}

The model updating approach described above is based on a parametric structural model. Health monitoring techniques may rely on nonparametric system identification approaches, in which a priori information about the natural of the model is not needed. Nonparametric models can be used to detect damage, although it is more difficult to use them for localization of damage. Among the nonparametric identification approaches that have been receiving growing attention recently are neural networks. Neural networks do not require information concerning the phenomenological nature of the system being investigated, and they also have fault tolerance, which makes them a useful means for representing model-unknown systems encountered in the real world. Neural networks do not require any prior knowledge of the system to be identified. It can treat both linear and nonlinear systems with the same formulation.

A number of investigators have evaluated the suitability and capabilities of these networks for damage detection purposes. Elkordy, et al. [lxv] used a finite-element model to develop failure 
patterns that were used to train a neural network so that it can later diagnose damage in the reference structure. Similar exploratory studies indicated that neural networks offer a powerful tool for assessing the condition of structures with inherent damage. But a study by Masri, et al. [lii] complements the work of other investigators by concentrating on a class of problems where knowledge of the failure states is not available. In other words, the potential failure modes of the test structure are so varied and so unpredictable that is not feasible to train the neural network by furnishing it with pairs of failure states and corresponding diagnostic response. By not postulating or searching among limited set of expected failure modes, the approach of this study can be applied equally well whether the underlying structural response is linear or not. However, such an approach has the disadvantage that detectable change in the signature of the analyzed response measure of the structure are not directly attributable to a specific failure mode, but simply indicate that damage has been sustained by an element or unit of a structure that has a dominant contribution to the response measure being analyzed.

Marwala [lxvi] presented a committee of neural networks technique, which employs frequency response function (FRF's), modal properties (natural frequencies and model shapes), and wavelet transformation (WT) data simultaneously to identify damage in structures. The committee approach assumed that the errors given by the three individual approaches were uncorrelated, a situation that became more apparent when using measured data rather than simulated data. The committee approaches were used in parallel to diagnose faults on a three-degree-of-freedom structure and a population of cylindrical shell. It is demonstrated that the committee procedure is more reliable than using each procedure individually. The disadvantage of the committee was that it required more than one trained network. The effectiveness of neural network methods is determined by the completeness of original data library and the reliability of algorithms. The neural network method may be effective for the on-line monitoring of large structures, such as cable-stayed bridges and suspension bridges.

\section{SENSORS AND OPTIMUM PLACEMENT}

One of the problems facing structural health monitoring is that very little is known about the actual stress and strains in a structure under external excitations. For example, the standard earthquake recordings are made of motions of the floors of the structure and no recordings are made of the actual stresses and strains in structural members. There is a need for special sensors to determine the actual performance of structural members.

Structural health monitoring requires integrated sensor functionality to measure changes in external environmental conditions, signal processing functionality to acquire, process, and combine multisensor and multi-measured information. Individual sensors and instrumented sensor systems are then required to provide such information.

\section{Sensor types}

Housner et al. [Error! Bookmark not defined.] summarized sensor types in structural control and their applications in civil engineering. In general, there are kinds of sensors for on-line monitoring, such as piezoelectric transducer, optical fiber sensors, embedded brag grating sensors, etc.. The reliability and durability of these sensors have being testified in many large bridges. Unlike many mechanical systems, typical bridge structures are often large in size and therefore have very low natural frequencies. In addition, the vibration level of the structural responses is very often quite. Therefore, the sensors of a monitoring system must be able to work in a very low frequency range and they must have a large dynamic measurement range. The industry has made great achievements in developing sensor and is still working forward. 


\section{Optimum Sensor Placement}

The estimation of the parameter values involves uncertainties due to limitations of the mathematical models used to represent the behavior of the real structure, the presence of measurement error in the data, and insufficient excitation and response bandwidth. In particular, the choice of the number and the location of the sensors in the structure have a major influence on the quality, or equivalently the uncertainty, of the model parameter estimation. Because complete modal data is impossible for a large flexible structure, measurements yield only partial mode shapes with respect to the total degree-of-freedom corresponding to the FEM, a common practice to bridge the gap is to expand the measured mode shapes or the reduction of freedom in FEM. Unfortunately, this process unavoidably introduces consequential errors and increases the difficulty in damage detection. One alternative method is to use the measured incomplete mode shapes to detect damage, then the relatively more information collected can be used advantageously for damage detection.

Methods have been developed to place sensors in an optimal fashion to address the identification and control of dynamic structures. Shi et al. [lx] presented a method in which the sensor locations were prioritized according to their ability to localize structural damage based on the eigenvector sensitivity method. Numerical examples and test results showed that this approach was effective for detecting structural damage directly using optimum and incomplete test modes.

The static and dynamic data are collected from all kinds of sensors which are installed on the measured structures. And these data will be processed and usable information will be extracted. So the sensitivity, accuracy, and locations, etc. of sensors are very important for the damage detections. The more information is obtained, the damage identification will be conducted more easily, but the price should be considered. That's why the sensors are determined in an optimal or near optimal distribution. In a word, the theory and validation of optimum sensor locations will still being developed.

\section{RESEARCH AND DEVELOPMENT NEEDS}

Most damage detection theories and practices are formulated based on the assumption: that failure or deterioration would primarily affect the stiffness and therefore affect the modal characteristics of the dynamic response of the structure. This is seldom true in practice, because of the following. First, traditional modal parameters (natural frequency, damping ratios and mode shapes, etc.) are not sensitive enough to identify and locate damage. The estimation methods usually assume that structures are linear and proportional damping systems. Second, most currently used damage indices depend on the severity of the damage, which is impractical in the field. Most bridge structures have redundancy in design and large in size with low natural frequencies. Any damage index should consider these factors. Third, scaled modeling techniques are used in current bridge damage detection. A single beam/girder models cannot simulate the true behavior of a real bridge. Similitude laws for dynamic simulation and testing should be considered. Fourth, many methods usually use the undamaged structural modal parameters as the baseline compared with the damaged information. This will result in the need of a large data storage capacity for complex structures. But in practice, there are majority of existing structures for which baseline modal responses are not available. Only one developed method [xl], which tried to quantify damage without using a baseline, may be a solution to this difficulty. There is a lot of research work to do in this direction. Fifth, seldom methods have the ability to distinguish the type of damages on bridge structures. To establish the direct relationship between the various damage patterns and the changes of vibrational signatures is not a simple work. 


\section{conclusions}

1. Unfortunately, nearly all the existing applications are based on developments and equipment's used in other engineering fields as in aerospace industries. The used instrumentation is usually expensive. Economical sensor placement and data collection methodologies, both onsite and remote, are needed in order for online health monitoring technologies to have practical benefits.

2. The reliability and durability of the entire health monitoring system should be studied. The interrelationship of structural behavior and the effect of each and all components of the monitoring system on the overall safety should be studied, including sensors and their optimal placements, communication, data acquisition, etc.

3. The evaluation of serviceability and load-carrying capacity for existing highway bridges based on the damage identification and reliability theory should be studied. It is very important for the load rating, condition assessment, and decision making of repair, strengthening, and rehabilitation of existing highway bridges.

\section{References}

1- Douglas, B.M., and W.H. Reid, “Dynamic Tests and System Identification of Bridges”, Journal of Structural Engineering, Vol. 108, No. 10, pp. 2295-2313: ASCE, 1982.

2- $\quad$ Salane, H.J., and J.W. Baldwin, “Identification of Modal Properties of Bridges”, Journal of Structural Engineering, Vol. 116, No. 7: ASCE, 1990.

i. $\quad$ Bakht, B. and Jaeger, L.G., "Bridge Testing-a Surprise Every Time", Journal of Structural Engineering, ASCE, Vol. 116, No. 5,pp. 605-611, 1990.

i. Kennedy, J.B. and Grace, N.F., "Prestressed Continuous Composite Bridges under Dynamic Load", Journal of Structural Engineering, ASCE, Vol. 116, No. 6, June, pp. 1660-1678, 1990.

i. Mazurek, D.P., and DeWolf, J.T., "Experimental Study of Bridge Health Monitoring Technique", Journal of Structural Engineering, ASCE, Vol. 116, No. 9, pp. 2532-2549, 1990.

i. Hearn, G., and Testa, RB., "Modal analysis for damage detection in structures", Journal of Structural Engineering, ASCE, Vol. 117, No. 10, pp. 3042-3063, 1991.

i. Houge, T.D., Aktan, A.E., and Hoyos, A., "Localized Identification of Constructed Facilities" Journal of Structural Engineering, ASCE, Vol. 117, No. 1, pp. 128-148, 1991.

i. Pandey, A.K., and Biswas, M., "Damage Detection in Structures Using Changes in Flexibility", Journal of Sound Vibration, Vol. 169, pp. 3-17, 1994.

i. $\quad$ Salawu, O.S., and William, C., "Bridge Assessment Using Forced-Vibration Testing", Journal of Structural Engineering, ASCE, Vol. 121, No. 2, pp. 161-172, 1995.

i. $\quad$ O.S. Salawu, “Assessment of Bridges: Use of Dynamic Testing”, Canadian Journal of Civil Engineering, Vol. 24, No. 2, pp. 218-228: National Research Council of Canada, 1997.

i. Salawu, O.S.; and C. Williams, "Theoretical and Experimental Vibration Analysis of a Reinforced Concrete Bridge", in Proceeding of the $15^{\text {th }}$ International Modal Analysis Conference, pp. 278-285, Florida: Society of Experimental Mechanics, 1997. 
i. $\quad$ Krishnan, K.; Seible, F.; and G. Pardoen, "Evaluation of Bridge Strengthening Measures Using Forced Vibration Tests”, in Proceeding of Structures Congress XIV: Building an International Community of Civil engineers, pp. 845-852, Chicago, Illinois: ASCE, 1996.

i. $\quad$ Farrar, C.R., and Jaurehui, D., "Damage Detection Algorithms Applied to Experimental and Numerical Modal Data from the 1-40 Bridge," Los Alamos National Laboratory, Report no. LA -13074 -MS, Los Alamos, New Mexico, 1996.

i. $\quad$ Doebling, S.W., Hemez, F.M., Peterson, L.D., and Farhat, C., "Improved Damage Location Accuracy Using Strain Energy-Based on Mode Selection Criteria", AIAA Journal, Vol. 35, pp. 693-699, 1997.

i. Kosmatka, I.B. and Rides, J.M., "Damage Detection in Structures by Modal Vibration Characterization", Journal of Structural Engineering, ASCE, Vol. 125, pp. 1384-1392, 1999.

i. $\quad$ Lenett, M.; Catbas, N.; Hunt, V.; Aktan, A.E.; Helmicki, A.; and D. Brown, "Issues in Multi-Reference Impact Testing of Steel-Stringer Bridges”, in Proceeding of the $15^{\text {th }}$ International Modal Analysis Conference, pp. 374-380, Florida: Society of Experimental Mechanics, 1997.

i. $\quad$ Catbas, F.N.; Lenett, M.; Brown, D.L.; Doebling, S.W.; Farrar, C.R.; and A. Turer, "Modal Analysis of Multi-Reference Impact Test Data for Steel Stringer Bridges”, in Proceeding of the $15^{\text {th }}$ International Modal Analysis Conference, pp. 381-391, Florida: Society of Experimental Mechanics, 1997.

i. Haritos, N.; and T.J. Chalko, "Using EMA to Determine the In-Service Condition of Bridges”, in Proceeding of the $15^{\text {th }}$ International Modal Analysis Conference, pp. 271-277, Florida: Society of Experimental Mechanics, 1997.

i. Sennah, K. and Kennedy, J.B., "Vibration of Horizontally Curved Continuous Composite Celluar Bridges", Canadian Journal of Civil Engineering, Vol. 25, 1998.

i. $\quad$ Chen, G.D, Yang, X.B, Alkhrdaji, T., et al., "Condition Assessment of Concrete Structures by Dynamic Signature Tests", in the Proceedings of the $13^{\text {th }}$ Engineering Mechanics Specialty Conference, ASCE, Baltimore, MD, N. Jones and R. Ghanem, Editors, June 1316, 1999.

i. Lee, G.C., and Liang, Z., "Development of a Bridge Monitoring System", in the Proceedings of the Second International Workshop on Structural Health Monitoring, Stanford University, Stanford, CA, pp. 349-358, 1999.

i. $\quad$ Aktan, A. E., Catbas, F.N., Turer, A., and Z.F. Zhang, "Structural Identification, Analytical Aspect", Journal of Structural Engineering, ASCE, 124, pp. 817-829, 1998.

i. Aktan, A.E., Tsikos, C.J., et al., "Challenge and Opportunities in Bridge Health Monitoring", in the Proceedings of the Second International Workshop on Structural Health Monitoring, Stanford University, Stanford, CA, 1999.

i. Huang, C.S., Yang, Y.B., Lu, L.Y., and Chen, C.H., "Dynamic Testing and System Identification of a Multi-span Highway Bridge", Earthquake Engineering and Structural Dynamics, Vol. 28, 1999.

i. Chaudhary, M.T., Abe, M., Fujino, Y., and Yoshida, J., "System Identification of Two Base-isolated Bridges using Seismic Records", Journal of Structural Engineering, ASCE, Vol. 126, pp. 1187-1195, 2000. 
i. Hwang, H, Jernigan, J.B., and Lin, Y.W., "Evaluation of Seismic Dynamic to Memphis Bridges and Highway Systems", Journal of Bridge Engineering, ASCE, Vol. 5, pp. 322330, 2000.

i. Haritos, N., "Dynamic Testing Techniques for Structural Identification of Bridge Superstructures", in the Proceedings of the International Conference on Advances in Structural Dynamics, edited by J.M. Ko and $\mathrm{Vol} \mathrm{Xu}$, The Hong kong Polytechnic University, Hong Kong, Dec., 2000.

i. $\quad$ Piombo, B.A., Fasana, A., Marchesiello, S. and Ruzzene, M., "Modeling and Identification of the Dynamic Response of a Supported bridge", Mechanical System and Signal Processing, Vol. 14, No. 1, 2000.

i. $\quad$ Kim, H.M., and Bartkowics, T.J., "An Experimental Study for Damage Detection using a Hexagonal Truss", Computer and Structures, Vol. 79, 2001.

i. Halling, M.W., Muhammad, I., and Womack, KC., "Dynamic filed testing for condition assessment of bridge bents", Journal of Structural Engineering, ASCE, Vol. 127, No. 2, 2001.

i. Peeters, B. and Roeck, G.D., "One-year Monitoring of the Z24-bridge: Environmental Effects versus Damage Events", Earthquake Engineering and Structural Dynamics, Vol. 30, 2001.

i. Salawu, O. S., "Detection of Structural Damage Through Changes in Frequency: a Review", Engineering Structures, Vol. 19, No. 9, 1997.

i. Doebling, S.W., Farrar, C.R, Prime, M.B., and Shevtiz, D.W., "A Review of Damage Identification Methods That Examine Changes in Dynamic Properties", Shock and vibration Digest, Vol. 30, 1998.

i. Zou, Y, Tong, L., and Steven, G.P., "Vibration-Based Model-Dependent Damage (Delamination) Identification and Health Monitoring for Composite Structures-a Review", Journal of Sound and Vibration, Vol. 230, No. 2, 2000.

i. Adams, R.D., Cawley, P., Pye, C.J., and Stone, BJ., "A Vibration Technique for Nondestructively Assessing the Integrity of Structures.", Journal of Mechanical Engineering Science, Vol. 20, 1978.

i. $\quad$ Biswas, M., Pandey, A.K., and Samman, M.M., "Diagnostic Experimental Spectral/Modal Analysis of a Highway Bridge", The International Journal of Analytical and Experimental Modal Analysis. Vol. 5, 1990.

i. Capecchi, D. and Vestroni, F., "Monitoring of Structural Systems by Using Frequency Data", Earthquake Engineering and Structural Dynamics, Vol. 28, 1999.

i. Casas, J.R, "Full-scale dynamic testing of the Alamillo Cable-Stayed Bridge in Sevilla", Earthquake Engineering and Structural Dynamics, Vol. 24, 1995, pp. 35-51.

i. Hearn, G., and Testa, RB., "Modal Analysis for Damage Detection in Structures", Journal of Structural Engineering, ASCE, Vol. 117, No. 10, 1991.

i. $\quad$ Stubbs, N., Kim, J.T., and Farrar, C.R., "Field Verification of a Nondestructive Damage Localization and Severity Estimation Algorithm", in the Proceedings of the $13^{\text {th }}$ International Modal Analysis Conference (IMAC), Nashville, TN, 1995. 
i. Pandey, AK., Biswas, M., and Samman, M.M., "Damage Detection from Changes in Curvature Mode Shapes", Journal of Sound Vibration, Vol. 145(2), 1991.

i. Wahab, M.M.A., Roceck, G.D., and Peeters, B., "Parameterization of Damage in Reinforced Concrete Structures using Modal Updating", Journal of Sound and Vibration, Vol. 228, No. 4, 1999.

i. Allemang, R.J.; and D.L. Brown, “A correlation Coefficient for Modal Vector Analysis”, in Proceeding of the $1^{\text {st }}$ International Modal Analysis Conference, pp. 110-116, Connecticut: Society of Experimental Mechanics, 1982.

i. Alampalli, S., Fu, G., and Aziz, I.A, " Modal Analysis as a Bridge Inspection Tool", in the Proceedings of the Tenth International Modal Analysis Conference (IMAC), pp. 1359-1366, San Diego, CA, 1992.

i. Alampalli, S., " Effects of testing, analysis, damage, and environment on modal parameters", Mechanical System and Signal Processing, Vol. 14(1), 2000.

i. Khalil, A. “Aspects in Nondestructive Evaluation of Steel Plate Girder Bridges”, Ph. D. Dissertation, Iowa State University, Iowa, USA, 1998.

i. Zhang, H., Schulz, M.J., and Ferguson, F., "Structural Health Monitoring Using Transmittance Functions", Mechanical System and Signal Processing, Vol. 13(5), pp. 765787, 1999.

i. Kim, H.M. and Bartkowicz, T.J., "A Two-Step Structural Damage Detection Approach with Limited Instrumentation", Journal of Vibration Acoustics, Vol. 119(2), 1997.

i. $\quad$ Kim, H.M. and Bartkowics, T.J., "An Experimental Study for Damage Detection Using a Hexagonal Truss", Computer and Structures, Vol. 79, 2001.

i. $\quad$ Koh, E.G., Hoon, B., and Liaw, C.Y., "Parameter Identification of Large Structural Systems in Time Domain", Journal of Structural Engineering, ASCE, Vol. 126, No. 8, pp. 957-963, 2000 .

i. Hao, H. and Xi A, Y, "Vibration-Based Damage Detection of Structures by Genetic Algorithm", Journal of Computing in Civil Engineering, ASCE, Vol. 16, No. 3, 2002.

i. Masri, S.P., Nakamura, M., et al., "A Neural Network Approach to the Detection of Changes in Structural Parameters", Journal of Engineering Mechanics, ASCE, Vol. 122, No. 5, 1996.

i. Masri, S.P., Smyth, AW., Chassiakos, AG., et al., "Application of neural network for detection of changes in nonlinear systems", Journal of Engineering Mechanics, ASCE, Vol. 126, No. 7, 2000, pp. 666-676.

i. $\quad$ Lin, J.W., Betti, R., Smyth, A.W., and Longman, R.W., "On-line Identification of Nonlinear Hysteretic Structural System Using a Variable Trace Approach", Earthquake Engineering and Structural Dynamics, Vol. 30, 2001.

i. Kerschen, G., and Golinval, J.C., "Theoretical and Experimental Identification of a Nonlinear Beam", Journal of Sound and Vibration, Vol. 244, No. 4, 2001.

i. Huang, C.H., "An Inverse Nonlinear Force Vibration Problem of Estimating the External Forces in a Damped System with Time-dependent System Parameters", Journal of Sound and Vibration, Vol. 242, No. 5, 2001. 
i. $\quad$ Lardies, J., and Larbi, N., "A New Method for Model Order Selection and Modal Parameter Estimation in Time Domain", Journal of Sound and Vibration, Vol. 245, No. 2, 2001.

i. Sohn, H., and Law, H.W., "Bayesian Probabilistic Damage Detection of a ReinforcedConcrete Bridge Column", Earthquake Engineering and Structural Dynamics, Vol. 29, 2000.

i. $\quad \mathrm{Xi}$ a, Y. and Hao, H., "Measurement Selection for Vibration-Based Structural Damage Identification", Journal of Sound and Vibration, Vol. 236, No. 1, 2000.

i. Shi, Z.Y, Law, S.S., and Zhang, L.M, "Improved Damage Quantification from Elemental Modal Strain Energy Change", Journal of Engineering Mechanics, ASCE, Vol. 128(5), 2002.

i. Wang, X., Hu, N., Fukunaga, H., and Yao, Z.H., "Structural Damage Identification Using Test Data and Changes in Frequencies", Engineering Structures, Vol. 23, No. 6, 2001.

i. Ren, W.X. and Roeck, G.D., "Structural Damage Identification Using Modal Data: I: Simulation Verification; II: Test Verification", Journal of Structural Engineering, ASCE, Vol. 128, No. 1, 2002.

i. Oh, B.H., and Jung, B.S., "Structural Damage Assessment with Combined Data of Static and Modal Tests," Journal of Structural Engineering, ASCE, 124(8), 1998.

i. Jang, H., Yeo, I., Shin, S., and Chang, S.P., "Experimental Investigation of SystemIdentification-Based Damage Assessment on Structures", Journal of Structural Engineering, ASCE , Vol. 128(5), 2002.

i. $\quad$ Elkordy, M.P., Chang, K.C, and Lee, G.C., "Neural Networks Trained by Analytically Simulated Damage States", Journal of Computing in Civil Engineering, ASCE, Vol. 7, No. 2, 1993.

i. Marwala, T., "Damage Identification Using Committee of Neural Networks", Journal of Engineering Mechanics, ASCE, Vol. 126, No. 1, 2000. 
